\title{
Empirical Analysis of the Impact of Heterogeneous Aging Families on Consumption based on CFPS Data
}

\author{
Yixuan Chen \\ School of Economics and Management, South east University, Nanjing, China \\ 824903158@qq.com
}

\begin{abstract}
Aging families are divided into with higher education and without higher education, and their influence on consumption is also different due to differences in their respective income characteristics. Relevant data based on CFPS 2014, 2016, and 2018 were empirical analyzed, and the results show that: (1) Aging families and consumption levels are negatively correlated, while the negative impact of aging families with higher education is even smaller. (2) Aging families have a negative correlation with subsistence, enjoyment, and developmental consumption, and their negative impact on subsistence consumption is the smallest, followed by developmental consumption, and enjoyment consumption is the largest. The aging families with higher education have less negative influence on various types of consumption than the other one. (3) In terms of regional differences, compared with the central and western regions, aging families in the eastern region, especially those with higher education in the eastern region, have a smaller negative impact on consumption levels. Among them, the most inhibitory effect on subsistence consumption is aging families without higher education in the central and western regions, the most inhibitory effect on enjoyment consumption is the aging families without higher education in the eastern region, and the most inhibitory effect on developmental consumption is the aging families without higher education in the central and western regions.
\end{abstract}

Keywords: Aging Families with Higher Education; Aging Families Without Higher Education; Consumption Level; Consumption Structure.

\section{Introduction}

Chinese population structure has undergone major changes in the past decade. The proportion of aging families has continued to rise, and aging families have increasingly become the focus of society. Compared with developed countries, China has entered an aging society later and faster. Aging families affect all aspects of society, such as consumption, industrial upgrading, and so on, which ultimately affect economic growth and have an important impact on the development of the country and society.

With the popularization of compulsory education, the proportion of the higher education population has increased. According to the China Statistical Yearbook, from 2014 to 2018, the proportion of higher education population in China increased from $10.59 \%$ to $14.58 \%$. The consumption concept of the higher education population has changed, which has a significant impact on consumption. At the same time, due to the demonstration effect, residents' consumption concepts will be in line with those of aging families with higher education, and aging families without higher education will have their consumption inputs affected by previous consumption due to the ratchet effect, which will realize the consumption level and consumption structure of continuous upgrading of a virtuous cycle.

In the new era of emphasizing the "new development pattern in which domestic and international double cycles promote each other with the domestic big cycle as the main body", consumption has a significant effect on the domestic cycle, and aging households are an important part of future consumption growth (Le Xin et al., 2016). The demonstration effect of aging families of higher education is conducive to further stimulating consumer demand and conducive to sustained and stable economic growth. However, previous studies have paid more attention to the influence of aging families or higher education level on consumption level and consumption structure respectively, ignoring the differences in the influence of heterogeneous aging families on consumption level and 
consumption structure. Therefore, we divide aging families into with higher education aging families and without higher education, exploring their respective effects on consumption and their mechanism of action, and conducting empirical analysis based on the relevant data of CFPS 2014, 2016, and 2018. It is of great significance for realizing the strategy of higher education development and promoting sustained and stable economic growth.

\section{Literature Review}

Western scholars have many relevant theories on consumption. The absolute income hypothesis put forward by Engel believes that absolute income is the main factor in determining consumption. Duesenberry believes that relative income determines consumption and includes two major effects: the demonstration effect and the ratchet effect. Modigliani's life cycle theory points out that income changes systematically in people's lives. Savings can make consumers transfer their income from a high-income work period to a low-income retirement period in order to smooth consumption.

Many scholars have conducted extensive research on the impact of aging households on consumption. The consumption of aging households is different from other consumers, there are certain characteristics, and there are huge internal differences (Faye Wong, 2015). There are three views on the impact of aging households on consumption levels. The first view is that aging households are conducive to raising household consumption levels. As aging families have more consumption needs, old age has become a stage of higher consumption levels, especially food expenditure and medical care expenditure (Le Xin et al., 2016). The second view is that aging households are not conducive to raising household consumption levels. For example, some researchers (Shi Beibei, 2017) found that the consumption level of aging households across the country is lower than the average consumption level through empirical research; He L et al. (He L et al., 2020) found that aging households have a negative impact on consumption level by constructing a model. The third view is that the relationship between aging families and family consumption levels is not significant (Yin Junru et al., 2016), because of the pension system and the lifestyle of parents and children live together. The consumption of aging families is smooth (Huang Yana et al., 2016). On the basis of the influence of aging households on the consumption level, scholars have conducted further research on the influence of aging households on the consumption structure. Aging households are closely related to the consumption structure. The consumption of non-durable goods, work-related expenditures, and food expenditures of aging households have decreased significantly, but the reduction in other consumption expenditures is not significant (Li Hongbin et al., 2015). Lin Xiaoshan (Lin Xiaoshan, 2018) found through the data analysis of the China Family Panel Studies (CFPS) that the more elderly people in aging households, the lower the consumption expenditure, especially for developmental consumption and enjoyment consumption, which is much lower than other types of households. Some scholars also found through data analysis of the China Health and Retirement Longitudinal Study (CHARLS) that aging households have different impacts on different consumption items (Zhao Xindong et al., 2018), and the consumption items that support jumping at different age nodes are different (Wei Jinrui et al., 2019). On this basis, some scholars have further studied the influence of aging households on consumption through regional comparison, and found that the influence of aging households on consumption in the central region is greater than that in the eastern and western regions (Qi Hongqian et al., 2020).

Regarding the effect of higher education level on consumption level, scholars have conducted research using the academic qualifications of the head of household and the highest academic qualifications of family members as proxy variables. There is a close relationship between the level of higher education and the level of consumption. Improving the level of higher education is conducive to raising the level of household consumption (Zhao Xindong et al., 2016; Wang Fang et al., 2019). Scholars have conducted further research on the impact of higher education level on consumption structure. The level of higher education has a significant positive impact on the consumption structure (Wu Shizi et al., 2018). Higher education is conducive to optimizing the 
consumption structure, and has a more significant impact on high consumption propensity and nonsubsistence consumer families (Di Junpeng et al., 2019). Specifically, the improvement of the level of higher education is conducive to increasing developmental consumption such as cultural consumption, luxury consumption, tourism consumption, and healthcare consumption (Li Guangming et al., 2019; Liu Yu et al., 2020; Wen Jiandong et al., 2019; Zhang Chao Et al., 2020; Cheah YK et al., 2020). On this basis, scholars have further studied the impact of higher education level on consumption through regional comparisons. Studies have found that the improvement of higher education has a more significant impact on the improvement of consumption levels in the eastern and central regions (Deng Taotao et al., 2020; Zhang Xuemin et al., 2018).

In summary, the existing literature provides a reference for the study of this article, but there are also the following shortcomings: First, the existing literature mostly studies the relationship between aging families or the level of higher education and consumption, but there are few studies on the impact of aging households with different education levels on consumption; Second, the existing studies are mostly national or individual provinces and cities. Therefore, the innovations of this article are as follows: divide aging families into aging families with higher education and aging families without higher education aging families two kinds of aging family evaluation system, qualitative analysis, and based on CFPS data of 2014, 2016 and 2018, empirical analysis was conducted to analyze the differences in the impact of heterogeneous aging households on consumption.

\section{The Impact Mechanism of Heterogeneous Aging Households on Consumption}

According to the absolute income hypothesis put forward by Engel, the income of aging families is greatly reduced due to the inability to participate in work, which leads to a reduction in consumption expenditure and a certain change in consumption structure. However, the above-mentioned effects will vary with the education level of aging families.

\subsection{The Relationship between Heterogeneous Aging Households and Consumption Levels}

\subsubsection{The Relationship between Aging Households in Higher Education and Consumption Levels}

Higher education has two transmission mechanisms for the role of consumption. One is intermediary transmission. Schultz's human capital theory points out that "education has the production function of increasing labor productivity and cultivating talents needed for economic development". Higher education is conducive to obtain more income, thereby increasing the level of consumption; the other is explained through behavioral economics, higher education is conducive to changing the concept of consumption, and subjectively has an impact on the level of consumption.

Because of higher labor productivity, aging families with higher education not only have more income in their youth, but also have higher retirement income in old age. Although consumption expenditures are lower, their consumption level is higher than that of aging families without higher education. On the other hand, according to the life cycle hypothesis, aging families can accumulate wealth through savings and consume wealth in old age to smooth consumption. As a result of higher education, aging families with higher education have changed their consumption concepts, and they pay more attention to the smoothing of consumption. They accumulate more wealth through savings in their youth, and their consumption levels are less affected by lower incomes in their old age.

\subsubsection{The Relationship between Aging Households Without Higher Education and Consumption Levels}

Since aging families without higher education have not received higher education, their income is lower not only in their youth, but also in retirement income in old age, so their consumption level will be lower than that of aging families with higher education. On the other hand, aging families without 
higher education have fewer concepts of smooth consumption, accumulate less wealth in their youth, and their consumption levels in old age are more affected by lower incomes.

\subsection{The Relationship between Heterogeneous Aging Families and Consumption Structure}

The consumption structure refers to the proportion of consumers who consume different consumption materials in a certain period. It can be divided into three categories: subsistence consumption, enjoyment consumption and developmental consumption. Among them, subsistence consumption includes consumption in food, clothing, housing and other aspects of consumption; enjoyment consumption includes consumption in household equipment and supplies, transportation and communication; developmental consumption includes consumption in culture, education, entertainment, and medical care.

Due to the decline in income of aging households, aging households have inhibitory effects on subsistence, enjoyment and developmental consumption expenditures, but they have different inhibitory effects on different types of consumption. Among them, aging households have the least inhibitory effect on subsistence consumption expenditure, followed by developmental consumption expenditure, and have the greatest inhibitory effect on enjoyment consumption expenditure. Following reasons: subsistence consumption is a rigid consumption demand. In the old age, aging families pay more attention to diet and health, and aging families have more leisure time, they can go shopping together, which has less inhibitory effect on subsistence consumption expenditure; the physical condition of aging families has declined, and they have more leisure time, so pay more attention to developmental consumption; aging families have purchased a lot of household equipment and daily necessities in their youth, and the physical condition of aging families has declined, travel is hindered, so enjoyment consumption expenses are limited.

\subsubsection{Differences in the Relationship between Aging Families in Higher Education and Consumption Structure}

Aging families with higher education have higher incomes and certain changes in their consumption concepts due to higher education. Although there is an inhibitory effect on various types of consumption, the inhibitory effect is less than that of aging families without higher education. With regard to subsistence consumption, aging families with higher education can purchase higher-quality food, clothing, shoes and hats because they have higher income and more savings, and the inhibitory effect is less than that of aging families without higher education. For enjoyment consumption, aging families with higher education may be willing to buy household equipment and daily necessities for their children, because they have more wealth. The physical condition allows a certain amount of travel due to they pay more attention to body maintenance during their youth, which can carry out a certain amount of transportation and communication consumption, and the inhibitory effect is less than that in aging families without higher education. With regard to developmental consumption, due to changes in the consumption concept of aging families with higher education, they pay more attention to body maintenance, and may undergo regular physical examinations and purchase health products. And they pay more attention to cultural consumption and conduct more cultural, educational and entertainment consumption, which inhibits less than aging families without higher education.

\subsubsection{Differences in the Relationship between Aging Families Without Higher Education and Consumption Structure}

Aging families without higher education have lower incomes because they have not received higher education. Consumption concept has not changed significantly, so the inhibitory effect on each type of consumption is greater than that of the aging families without higher education. For subsistence consumption, aging families without higher education have less income and savings, and may tend to have light diet and plain dress, which has a greater inhibitory effect than aging families with higher education. With regard to enjoyment consumption, aging households without higher education have less wealth, less purchase of household equipment and daily necessities, less consumption of transportation and communication due to physical limitations, and the inhibitory 
effect is greater than that of aging households with higher education. For developmental consumption, since the consumption concept of aging families without higher education has not changed significantly, consumption of health care may be more limited to public medical care, and the consumption of culture, education and entertainment is not very important, and the inhibitory effect is greater than that of aging families with higher education.

In addition, there are certain regional differences in the relationship between aging households and consumption levels and consumption structure. Due to the higher level of economic development and better social security services in the eastern region, the living standards of aging families are higher, and the level of consumption decline is less than that of the central and western regions. As a result of receiving higher education, aging families with higher education have more retirement income and savings, and their consumption level declines less than that of aging families without higher education. It can be seen that the aging households with higher education in the eastern region have the least negative impact on consumption levels.

Although aging households have an inhibitory effect on subsistence, enjoyment and developmental consumption expenditure due to the decline in their income level, they have different restraints on different types of consumption. (1) For the subsistence consumption, the aging families without higher education in the central and western regions have a greater inhibitory effect. Due to the low level of economic development in the central and western regions, the social security system is not perfect, and the living standard of aging families is low, which has a great inhibitory effect on subsistence consumption. Secondly, due to the lack of higher education, the aging families without higher education have less income and savings, and their consumption concept has not changed significantly, which has a greater inhibitory effect on subsistence consumption. (2) For enjoyment consumption, aging families without higher education in the eastern region have a greater inhibitory effect. Due to the higher level of economic development in the eastern region and more complete social security services, aging families can travel for free when they meet certain conditions, which has a greater inhibitory effect on enjoyment consumption. Secondly, the income and savings of aging families without higher education are small due to have not received higher education and they may not buy household equipment and daily necessities for their children, which has a greater inhibitory effect on enjoyment consumption. (3) For developmental consumption, aging families without higher education in the central and western regions have a greater restraint. Due to the low level of economic development in the central and western regions, the social security system is not perfect, and the living standards of aging families are low. After meeting their basic living needs, there is less demand for developmental consumption, which has a greater inhibitory effect on developmental consumption. Secondly, aging families without higher education have not received higher education, have less income and savings, and have not changed much in their consumption concepts. They pay less attention to developmental consumption, so that it has a greater inhibitory effect on developmental consumption.

\section{Empirical Analysis of Consumption by Heterogeneous Aging Families}

\subsection{Model Setting}

Since this article hopes to examine the impact of heterogeneous aging households on consumption in the context of aging, the following economic model is established (Wang Wei et al., 2017) based on the methods of existing literature:

\subsubsection{Consumption Level Model}

lnpce ${ }_{i t}=\alpha 1+\beta 1$ ratio_old ${ }_{i t}+\delta X_{i t}+\varepsilon_{i t}$

Among them, i represents family; $t$ represents time (unit: year); lnpceit represents the resident consumption expenditure (unit: yuan) of the i-th family in t years, which is the consumption level referred to in this article. 


\subsubsection{Consumption Structure Model}

Engels divided the living materials into subsistence materials, enjoyment materials and development materials, which has become an important method for measuring consumption structure (1) so far.

This article draws on the practice of Lin Xiaoshan (Lin Xiaoshan, 2018), using food, clothing, and housing consumption expenditure to measure subsistence consumption; we use household equipment and supplies, transportation and communication consumption expenditure to measure enjoyment consumption; and we use culture, education, entertainment, and healthcare consumption expenditure to measure developmental consumption. In order to investigate the impact of aging families on the consumption structure, this article constructs the regression equations of population aging and subsistence, enjoyment and developmental consumption expenditures:

Subsistence consumption model: Insubsistence ${ }_{i t}=\alpha_{2}+\beta_{2}$ ratio_old ${ }_{i t}+\delta X_{i t}+\varepsilon_{i t}$

Enjoyment consumption model: Inenjoyit $=\alpha_{3}+\beta_{3}$ ratio_old ${ }_{i t}+\bar{\delta} X_{\text {it }}+\varepsilon_{\text {it }}$

Developmental consumption model: Indevelopment ${ }_{\mathrm{it}}=\alpha_{4}+\beta_{4}$ ratio_old $_{\mathrm{it}}+\delta \mathrm{X}_{\mathrm{it}}+\varepsilon_{\mathrm{it}}$

Among them, lnsubsistence, lnenjoy, and lndevelopment respectively represent the subsistence, enjoyment and developmental consumption expenditures of aging families (unit: yuan). The subsistence, enjoyment, and developmental consumption expenditures of aging households are obtained through the sum of consumption items, and they are measured in logarithmic form.

ratio_old is the core explanatory variable of this article. It represents the proportion of the elderly in the family. Drawing on the traditional United Nations standard, it is measured by the proportion of elderly people aged 60 and over in the total population in the family. It is called an aging family if the proportion is greater than $10 \%$. In addition, this article combines the practice of the existing literature and uses the highest education level among family members as the proxy variable of the human capital level of the entire family (Di Junpeng et al., 2019). It is called a higher education family if the highest education level among family members is a college degree or above (Wu Shizi et al., 2018).

$\mathrm{X}$ represents a series of control variables, combined with the existing literature, the control variables in this article are: (1) Per capita household income (unit: yuan), measured in logarithmic form. According to the absolute income hypothesis, generally, the higher the per capita income, the higher the consumption expenditure; (2) The proportion of males and females in the household, measured by the proportion of the number of males and females in the household in the total household population. Generally, male consumption behaviors are more rational, and the higher the proportion of males in the household, the lower the consumption expenditure; (3) The number of people whose work units are farmers or households in the family (unit: person). Generally, households are usually more willing to consume than farmer households. The regression coefficient value of the population number of the farmer households is expected to be negative, and the regression coefficient value of the population number of the households is expected to be positive; (4) The number of people with pension insurance in the family (unit: person). Pension insurance provides protection for the consumption behavior of the elderly population, and the regression coefficient value is expected to be positive; (5) The number of people inside and outside the system (unit: person) in the family, usually the consumption expenditures of the population inside and outside the system are similar, and the impact on the consumption structure needs to be further tested.

${ }^{(1)}$ See the anthology of marne, vol. 1, p. 349.

\subsection{Data Sources and Descriptive Statistics}

The data used in this article comes from the baseline survey data of China Family Panel Studies (CFPS) in 2014, 2016, and 2018. The CFPS sample covers 25 provinces, cities, and districts across the country, encompassing $95 \%$ of the national population. The research in this article mainly involves the age, education, income and consumption of family members in the CFPS survey. In the process of data processing, first extract the data of household income, consumption expenditure and other data in the CFPS 2014, 2016, and 2018 household databases, as well as the data such as age and 
education in the personal database, and merge the household and personal databases to form a new database, and calculate the proportion of elderly people aged 60 and over in the total population, identifying the highest level of education in the family, etc. Then delete the households in which the proportion of the elderly aged 60 and above in the total population is less than $10 \%$, and finally we get a national sample of 26,594 households. Among them, there are 1,2003 households in the eastern region and 14,586 households in the central and western regions. There are 4903 households with the highest education level of college and above, and 21689 households with high school and below.

The descriptive statistics of the main variables are shown in Table 1. Table 1 shows that the average annual consumption expenditure of residents taking the logarithm is 10.3248 , of which the average annual consumption of the subsistence type is 9.7169, the average annual consumption of the enjoyment type is 8.2530 , and the average annual consumption of the development type is 8.4754. The average annual consumption expenditure of residents of aging families with higher education is significantly higher than that of aging families without higher education. The average annual consumption for subsistence of aging families with higher education is higher than that of aging families without higher education. And the average annual consumption of enjoyment type and development type are significantly higher than that of aging families without higher education. Secondly, the standard deviation of consumption expenditure of aging families with higher education is smaller than that of without higher aging families. It can be seen that the impact of heterogeneous aging families on consumption varies significantly.

\subsection{Benchmark Regression Results and Analysis}

This article first uses the OLS method to regress the influence of the degree of aging families on the consumption level, and analyzes the consumption of subsistence, enjoyment and development respectively. The specific regression results are shown in Table 2 . The regression coefficients of the test results are significant at the 1\% significance level, and the degree of fit is good, which proves that the settings of each model are reasonable and effective. The main findings of our empirical results are as follows:

\subsubsection{Impact on Consumption Levels}

In the regression model of column (1) in Table 2, the regression coefficient of the degree of aging families is significantly negative at the $1 \%$ significance level, and the coefficient of elasticity is 0.503, indicating that among aging families with higher education, the higher the degree of aging families, the higher the proportion of the elderly in the household, the lower the consumption expenditure of residents, and the lower the consumption level. In the regression model of column (5) in Table 32, the regression coefficient of the degree of aging families is significantly negative at the $1 \%$ significance level, and the elasticity coefficient is -0.986 , indicating that among the aging families without higher education, the higher the degree of aging families, the greater the inhibition effect on consumption. In summary, the impact of aging families on the consumption level is significantly negative. The reason is that as the degree of aging families increases, the income of the elderly decreases, thus reducing consumption expenditures. In contrast, the negative impact of aging families with higher education is smaller. The reason is that aging families with higher education receive higher education, and they tend to be more inclined to smooth consumption, so the inhibitory effect on consumption is smaller.

\subsubsection{Impact on Consumption Structure}

In the regression model of columns (2) and (6) in Table 2, the regression coefficient of the degree of aging families is significantly negative at the $1 \%$ significance level, and the elastic coefficients are -0.261 and -0.827 , indicating that the higher the degree of aging families, the subsistence consumption expenditure is lower, because as the degree of aging families increases, the income of the elderly decreases, so the subsistence consumption expenditure is reduced. The regression results also show that aging families without higher education have a greater inhibitory effect on subsistence consumption. The reason is that aging families with higher education are more inclined to smooth 
consumption due to they have received higher education, so the inhibition effect on subsistence consumption is smaller.

In the regression model of columns (3) and (7) in Table 2, the regression coefficient of the degree of aging families is significantly negative at the $1 \%$ significance level, and the elastic coefficients are -1.540 and -1.554 , indicating that the higher the degree of aging families, the enjoyment consumption expenditure is lower, because as the degree of aging families increases, the income of the elderly decreases, so the enjoyment consumption expenditure is reduced. The regression results also show that aging families without higher education have a greater inhibitory effect on enjoyment consumption. The reason is that aging families with higher education tend to be more inclined to smooth consumption due to higher education, and therefore they have a smaller inhibitory effect on enjoyment consumption.

In the regression model of columns (4) and (8) in Table 2, the regression coefficient of the degree of aging families is significantly negative, and the elastic coefficients are -0.430 and -1.000 , indicating that the higher the degree of aging families, the lower the developmental consumption expenditure. The reason is that as the degree of aging households increases, the income of the elderly decreases, thus reducing developmental consumption expenditures. The regression results also show that aging families without higher education have a greater inhibitory effect on developmental consumption. The reason is that aging families with higher education tend to be more inclined to smooth consumption due to higher education, and have a smaller inhibitory effect on developmental consumption.

In addition, although the degree of aging families has a significantly negative impact on subsistence, enjoyment, and developmental consumption, it has the least inhibitory effect on subsistence consumption, followed by developmental consumption, and enjoyment consumption is the largest. The reason is that subsistence consumption is rigid consumption demand. Even if the degree of aging households increases, the inhibitory effect on subsistence consumption is limited. With the increase in the degree of aging families, the older people have more leisure time, and the inhibitory effect on cultural, educational and entertainment consumption is limited; The physical health of the elderly is declining, and the inhibitory effect on health care consumption is limited, so the inhibitory effect on developmental consumption is limited. Due to the increase in the degree of aging families, older people tend not to purchase household equipment and supplies, and the consumption of household equipment and supplies decreases; due to travel difficulties, transportation and communication consumption decreases, so the inhibitory effect on enjoyment consumption is the most significant.

In summary, the degree of common aging families has a significantly negative impact on subsistence, enjoyment, and developmental consumption, and in aging families without higher education, the inhibitory effect on various types of consumption is greater.

\subsubsection{Other Findings}

The impact of per capita net household income on consumption is significantly positive, in line with Keynesian consumption theory. The negative impact of men on consumption is significantly greater than that of women. This may be because men's consumption behaviors are more rational and reduce more non-essential consumption. The number of people whose work unit is a farm household has a negative impact on consumption, and the number of of people whose work unit is a household has a positive impact on consumption. The impact of pension insurance on consumption is significantly positive, and the positive impact on the developmental consumption of aging families with higher education is the most significant. This may be because the elderly have security and are more willing to consume, and the elderly with higher education hope to further improve their educational level and physical health status, and pay more attention to consumption of culture, education, entertainment and medical care consumption. The number of people inside and outside the system has a positive impact on consumption. 


\subsection{Regression Results and Analysis by Region}

We have also returned to the eastern region and the central and western regions to examine the regional differences in the impact of heterogeneous aging families on consumption. The specific regression results are shown in Table 3 and Table 4. Our main findings are:

\subsubsection{Impact on Consumption Levels}

In the regression model of column (1) in Table 3, the regression coefficient of the degree of aging households is significantly negative at the $1 \%$ significance level, and the elasticity coefficient is 0.359. In the regression model of column (5) in Table 3 , the regression coefficient of the degree of aging households and the regression coefficient is significantly negative at the $1 \%$ significance level, and the elasticity coefficient is -0.934 , indicating that the higher the degree of aging families, the lower the consumption level, and in aging families without higher education, the inhibitory effect on consumption is greater. In the regression model of column (1) in Table 4 , the regression coefficient of the degree of aging families is significantly negative at the $1 \%$ significance level, and the elasticity coefficient is -0.618 . In the regression model of column (5) in Table 4 , the regression coefficient of the degree of aging families is significantly negative at the significance level of $1 \%$, and the elasticity coefficient is -1.033 , indicating that the higher the degree of aging families, the lower the consumption level, and in aging families without higher education, the inhibitory effect on consumption is greater. In summary, the degree of aging families has a significantly negative impact on the consumption level. The reason is that as the degree of aging families increases, the income of the elderly decreases, thus reducing consumption expenditures. However, the negative impact of aging families with higher education in the eastern region is smaller, because the economic development level in the eastern region is higher, and the living standards of residents are higher. Therefore, the degree of aging families has less negative impact on consumption levels; due to the higher education, aging families tend to consume more smoothly and have less negative influence on consumption level.

\subsubsection{Impact on Consumption Structure}

In the regression model of columns (2) and (6) in Table 3, the regression coefficient of the degree of aging households is negative, and the elasticity coefficients are -0.144 and -0.819 . In the regression model of columns (2) and (6) in Table 4, the regression coefficient of the degree of aging households is significantly negative, and the elastic coefficients are -0.423 and -0.867 , both of which indicate that the higher the degree of aging families, the lower the subsistence consumption expenditures. The reason is that as the degree of aging families increases, the income of the elderly decreases and make the subsistence consumption expenditure lower. The regression results also show that the aging families without higher education in the central and western regions have the greatest inhibitory effect on subsistence consumption. The reason is that the eastern region has a higher level of economic development and higher living standards, so the inhibitory effect on subsistence consumption is smaller; aging families with higher education tend to be more inclined to smooth consumption because they receive higher education, so the inhibitory effect on subsistence consumption is smaller.

In the regression model of columns (3), (7) in Table 3, the regression coefficient of the degree of aging families significantly negative at the $1 \%$ significance level, and the elastic coefficients are 1.432 and -1.536. In the regression model of columns (3), (7) in Table 4, the regression coefficient of the degree of aging families is significantly negative at the $1 \%$ significance level, and the elastic coefficients are -1.478 and -1.504 , indicating that the higher the degree of aging households, the lower the enjoyment consumption expenditure. The reason is that, as the degree of aging households increases, the income of the elderly decreases, thus reducing enjoyment consumption expenditures. However, among aging families without higher education in the eastern region, the inhibitory effect on enjoyment consumption is the greatest. This may be due to the higher economic development level and social security level in the eastern region, the travel of aging families is concessionary, so enjoyment consumption expenditure is less; aging households without higher education have lower income and savings levels and lower enjoyment consumption expenditures because they have not received higher education. 
In the regression model of columns (4) and (8) in Table 3, the regression coefficient of the degree of aging families is negative, and the elastic coefficients are -0.471 and -0.845 . In the regression model of columns (4) and (8) in Table 4, the regression coefficient of family level is negative, and the elasticity coefficients are $-0.277,-1.116$, indicating that the higher the degree of aging families, the lower the developmental consumption expenditures. The reason is that as the degree of aging families increases, the income of the elderly decreases and therefore decreases developmental consumption expenditure. However, aging families without higher education in the central and western regions have the greatest inhibitory effect on developmental consumption. This may be due to the eastern region has a higher level of economic development and a higher standard of living, so the inhibitory effect on developmental consumption is smaller; aging families with higher education tend to be more inclined to smooth consumption as they have received higher education, so the inhibitory effect on developmental consumption is smaller.

In summary, the degree of aging families has a significantly negative impact on subsistence, enjoyment, and developmental consumption. However, the most inhibitory effect on subsistence consumption is aging families without higher education in the central and western regions, the most inhibitory effect on enjoyment consumption is the aging families without higher education in the eastern region, and the most inhibitory effect on developmental consumption is the aging families without higher education in the central and western regions.

\subsubsection{Other Findings}

The regression results of other control variables are similar to Table 2.

\section{Conclusions and Policy Recommendations}

This paper uses the balanced panel data of CFPS 2014, 2016, and 2018, based on the social background of aging, and focuses on the impact of heterogeneous aging households on consumption. The conclusions are as follows: (1) In terms of the impact on consumption level, the degree of aging families has a significantly negative impact on consumption levels and the negative influence of aging families with higher education is even smaller. (2) In terms of the impact on the consumption structure, the degree of aging families has a significantly negative impact on subsistence, enjoyment, and developmental consumption, but aging families without higher education have a greater inhibitory effect on various types of consumption. Although the degree of aging families has a significantly negative impact on subsistence, enjoyment, and developmental consumption, it has the least inhibitory effect on subsistence consumption, followed by developmental consumption, and enjoyment consumption is the largest. (3) In terms of regional differences, the degree of aging families has a significantly negative impact on consumption levels, but in the eastern region, especially in aging families with higher education in the eastern region, negative impact is less. The degree of aging families has a significantly negative impact on subsistence, enjoyment, and developmental consumption. However, the most inhibitory effect on subsistence consumption is aging families without higher education in the central and western regions, the most inhibitory effect on enjoyment consumption is the aging families without higher education in the eastern region, and the most inhibitory effect on developmental consumption is the aging families without higher education in the central and western regions.

First, improve family planning and retirement policies to delay the aging process. For all regions, the degree of aging households has a significantly negative impact on consumption levels. As the income of the elderly people after retirement has decreased significantly, consumption expenditures need to be reduced, which is not conducive to sustained and stable economic growth. Therefore, on the one hand, the government needs to improve the family planning policy and reduce the proportion of the aging population by encouraging childbirth; on the other hand, the government needs to improve the retirement policy, appropriately delay the retirement age, increase the number of years for the aging population to obtain stable income, and delay the aging process. 
Second, improve the compulsory education system and raise the level of education. Although the degree of aging families has a significantly negative impact on consumption levels, the negative impact of aging families with higher education is even smaller. Therefore, on the one hand, the government needs to increase investment in education to provide assistance to students from poor families and ensure that students receive compulsory education; on the other hand, the government needs to encourage primary and secondary schools to provide teachers with more preferential salaries to ensure the quality of compulsory education.

Third, develop aging industries to meet consumer demand. Although the degree of aging families has a significantly negative impact on subsistence, enjoyment, and developmental consumption, it has the least inhibitory effect on subsistence consumption, followed by developmental consumption, and enjoyment consumption is the largest. Subsistence consumer demand is the rigid consumer demand of the aging population, which is already in a saturated state, while development consumer demand exceeds supply, which can become a new economic growth point.

From the perspective of health care consumption, on the one hand, the government can cooperate with medical institutions to encourage aging families to undergo regular physical examinations at designated hospitals to stimulate the consumption demand of aging families; on the other hand, medical institutions need to improve the level of diagnosis and treatment through high-quality products and services to meet the consumer needs of aging families. From the perspective of cultural, educational and entertainment consumption, on the one hand, the government can encourage the development of the cultural, educational and entertainment industry through tax cuts and other policies, and meet the consumption needs of aging families through the establishment of universities for the elderly. On the one hand, enterprises need to push through the old and bring forth the new. On the basis of fully understanding the consumption needs of aging families, enterprises should develop products suitable for aging families and cater to their consumption needs.

Fourth, vigorously develop the economic and social security system and increase consumer demand. The empirical results found that pension insurance has a significant positive impact on consumption. Therefore, it is necessary to improve the social security system to stimulate the consumption demand of aging families. On the one hand, the government needs to increase the proportion of social security expenditures, provide more comprehensive social security services, and provide confidence in the consumption of aging families through social security services involving medical care, elderly care, and nursing care; on the other hand, enterprises need to pay attention to social security issues, have more social care, and provide employees with reasonable pensions. Through the cooperation between the government and enterprises, it will provide protection for the consumption of aging families, increase the consumption demand of aging families, and promote economic growth.

Compared with the central and western regions, the eastern region has a more complete social security system. Therefore, the government needs to pay attention to the economic development of underdeveloped regions and reduce regional differences. First, realize a more reasonable fiscal transfer payment system and increase transfer payment expenditures in the central and western regions; Second, realize a more reasonable tax system, levy more taxes in the eastern region, levy less taxes in the central and western regions, and encourage funds in the eastern region flow to the central and western regions. Promote the economic development of the central and western regions through reasonable policies and measures, and increase the consumption demand of aging families in the central and western regions.

\section{References}

[1] Cheah Y K, Adzis A A, Bakar J A B U, et al. 2020 Age, education and consumption of medical care: evidence from malaysia[J]. Malaysian Journal of Public Health Medicine, 2020, 20(1): 109-121.

[2] Cheng Z. 2021 Education and consumption: Evidence from migrants in Chinese cities[J]. Journal of Business Research, 2021, 127: 206-215. 
[3] He L, Zhou S, Liu Z. 2020 How is aggregate household consumption affected jointly by longevity, pension, and aging? Theory and evidence[J]. International Review of Economics, 2020, 67(4): 499-512.

[4] Wang M, Yu X. 2020 Will China's population aging be a threat to its future consumption? [J]. China Economic Journal, 2020, 13(1): 42-61.

[5] Deng Taotao, Hu Yukun, Yang Shengyun, Ma Mulan. 2020 Rural household income sources, household characteristics and tourism consumption: A micro analysis based on the data of Chinese Household Tracking Survey (CFPS) [J]. Tourism Journal, 2020, 35(01): 47-62.

[6] Di Junpeng, Yuan Yan, Zhang Xinyue. 2019 Education level, consumption propensity and consumption structure--An empirical analysis based on quantile regression [J]. Shanghai Economic Research Journal, 2019(02): 58-66.

[7] Huang Yana, Wang Tianyu. 2016 Will retirement affect consumption? --Evidence from China's Transition [J]. World Economic Review,2016(01):87-107.

[8] Le Xin, Peng Xizhe. 2016 Journal of Fudan University (Social Sciences Edition), 2016, 58(02): 126-134.

[9] Li G M, Xu Y. 2019 Income, time and knowledge: the constraining effect of cultural consumption capacity [J]. University, 2019 (6): 106-113.

[10] Li Hongbin, Shi Xinzheng, Wu Binzhen. 2015 Research on the consumption behavior of Chinese residents before and after retirement [J]. Economics Quarterly, 2015, 14(01): 117-134.

[11] Lin Xiaoshan. 2018 Family aging, consumption structure and consumption stratification -- data analysis based on CFPS2012 [J]. Journal of Southeast University (Philosophy and Social Sciences Edition), 2018, 20 (2): 112-121.

[12] Liu Yu, Zhou Jianxin. 2020 Spatial differences and driving factors of cultural consumption in China [J]. Statistics and Decision Making, 2020, 36(13): 90-93.

[13] Qi Hongqian, Liu Yan. 2020 Changes in population age structure and upgrading of household consumption: an empirical study based on CFPS data [J]. China Population, Resources and Environment, 2020, 30(12):174-184.

[14] Shi Peipei. 2017 An empirical study on the consumption of the elderly population in urban and rural China -- also on the "retirement-consumption puzzles" [J]. Population Research,2017,41(03):53-64.

[15] Shi Mingming, Jiang Zhou, Qiu Xurong. 2019 How aging affects household consumption expenditure in China: evidence from China comprehensive social survey [J]. Economic Theory and Management, 2019 (04): $62-79$.

[16] Tian Hui. 2013 Consumption economics [M]. Shanghai: Tongji University Press, 2013:2-10.

[17] Wang Wei, Liu Yufei. 2017 Population aging and the upgrading of household consumption structure -an empirical study based on CFPS2012 data [J]. Journal of Shandong University (Philosophy and Social Sciences Edition), 2017(05): 84-92.

[18] Wang Fang, Huang Lifang. 2019 Analysis of the impact of household characteristics on consumer expenditure: based on Chinese household tracking survey data [J]. Journal of Mathematical Statistics and Management, 2019, 38(03): 381-393.

[19] Wang F. 2015 An empirical study on the consumption behavior of the urban elderly in China [J]. Population and Development, 2015, 21(03): 101-112.

[20] Wei Jinrui, Zhang Ruiling. 2019 Aging and the consumption behavior of the elderly family [J]. statistical research, 2019, 36(10): 87-99.

[21] Wen Jiandong, Cai Zhiquan. 2019 Research on the difference of luxury buying behavior of heterogeneous consumer groups based on CHFS data [J]. Research on Financial Issues, 2019(09): 128-136.

[22] Wu Shiying, Ma Mang. 2018 Population changes, consumption structure and residents' consumption potential release: GMM analysis based on provincial dynamic panel data [J]. Contemporary Economic Management, 2018, 40(04): 8-15.

[23] Yin Junru, Xu Haoyi, Ni Xuanming. 2016 Research on the impact of population aging on residents' consumption level: theoretical analysis and empirical test based on optimal growth model [J]. Systems Engineering Theory \& Practice, 2016, 36(12): 3034-3045. 
[24] Zhang Chao, Yan Jianye, Kang Jian. 2020 Reading ten thousand books, traveling ten thousand miles -the influence of educational level on tourism consumption [J]. Technology Economics, 2020, 39(08): 114118.

[25] Zhang Xuemin, Shen Liyuan. 2018 How does education affect consumption? Journal of Southwest University (Social Science Edition), 2018, 44(04): 100-111+194-195.

[26] Zhao Xindong, Li Lin. 2016 How household economic factors and population characteristics affect consumption of urban residents with different income levels [J]. Mathematical Statistics and Management, 2016, 35(06): 1076-1085.

[27] Zhao Xin-Dong, Wang Hao. 2018 The impact of retirement on household consumption: based on fuzzy breakpoint regression [J]. Journal of Wuhan University (Philosophy and Social Sciences Edition), 2018, 71 (01): 167-174. 